\title{
Phase Noise Jitter Synchronization for Coherent Optical OFDM via Pilot-Data-Aided and Wiener Filter
}

\author{
Jean Temga ${ }^{1}$, Liu Deming ${ }^{1}$, Mahamadou Hamidine ${ }^{2}$, Zhang Minming $^{1}$ \& Carine H. Maiawe ${ }^{3}$ \\ 1 National Engineering Laboratory for Next Generation Internet Access System, School of Optical and \\ Electronics Information Engineering, Huazhong University of Science and Technology, Wuhan, China \\ ${ }^{2}$ Wuhan National Laboratory of Optoelectronic, School of Optical and Electronic Information Engineering, \\ Huazhong University of Science and Technology, Hongshan, Wuhuan, Hubei, China \\ ${ }^{3}$ Department of Computer Science and Telecommunications, Higher institute of Sahel, University of Maroua, \\ Cameroon
}

Correspondence: Jean Temga, National Engineering Laboratory for Next Generation Internet Access System, School of Optical and Electronics Information Engineering, Huazhong University of Science and Technology, Wuhan 430074, China. Tel: 86-159-2705-4167. E-mail: jeantemga@gmail.com

\author{
Received: February 15, $2014 \quad$ Accepted: March 25, $2014 \quad$ Online Published: April 22, 2014 \\ doi:10.5539/cis.v7n2p56 \\ URL: http://dx.doi.org/10.5539/cis.v7n2p56
}

\begin{abstract}
We investigate a carrier phase jitter synchronization technique for square M-ary quadrature amplitude modulation (M-QAM) coherent optical orthogonal frequency division multiplexing (CO-OFDM) signal employing a unique pilot's system design, Feed forward maximum likelihood phase estimator as well as Wiener filter-type Minimum Mean square error (MMSE) interpolator. The wiener filter relies upon Kolmogorov type to interpolate the estimated phase noise with $\mathrm{M}$ taps.

A $20 \mathrm{~Gb} / \mathrm{s}$ CO-OFDM via 4-QAM, 16-QAM, 64-QAM then 256-QAM modulation is applied as simulation model in Optisystem. System efficiency is evaluated throughout phase root mean square error (RMSE) calculated in degree. A comparative investigation of four different modulation techniques found that 4-QAM performs with good RMSE versus the rest of square M-QAM. A free-noise receiver, a pilot aided feed forward maximum likelihood (PA-FF-ML) receiver and a PA-FF-ML with MMSE (PA-FF-ML-MMSE) are compared. PA-FF-ML-MMSE exhibited superior performance rather than receiver using just PA-FF-ML.
\end{abstract}

Keywords: coherent optical OFDM, local oscillator laser, pilots data aided, MMSE, M-QAM

\section{Introduction}

To meet the rising interest in the capacity and data-rate requirements in optical communication systems, CO-OFDM systems have captivated a vast involvement in recent years (Shieh, 2008). This is certainly simply because it offers the following features; high spectral efficiency in electrical in addition to optical domain, dispersion insensitivity, as well as computation efficiency (Shieh \& Athaudage, 2006). In contrast, the major issue of CO-OFDM is the fact that the phase noise of the local oscillator and sampling frequency needs to be compensated for, since these problems cause it to be vulnerable to synchronization errors compared to the single-carrier method (Pollet, Van Bladel, \& Moeneclaey, 1995; Shieh \& Athaudage, 2006). In previous research (Minming, Deming, \& He, 2012), it was indicated that frequency clock errors and local oscillator laser phase noise are the causes of phase jitter in CO-OFDM system. Consequently, details information of phase jitter synchronization in CO-OFDM signals deserve careful attention. In conventional CO-OFDM systems, there are three steps of CO-OFDM synchronization (Shieh \& Djordjevic, 2009): timing synchronization, frequency synchronization, and subcarrier recovery. The first couple of steps are usually carried out in the time domain, whilst the last step performs in the frequency domain.

Schmidl and Cox (Schmidl \& Cox, 1997) made use of correlation property of periodical training symbols for timing and frequency estimation. In this particular approach, timing metric carries a plateau, resulting in a large variance in symbol timing estimation.

In the same sense, Minn proposed a similar method with a sharper timing metric to reduce the uncertainly of timing estimation (Minn, Bhargava, \& Letaief, 2003), but the estimation variance remains large in the dispersive 
channels. An impulse-like shape at the correct symbol timing point to correct Minn method was proposed by Park (Park, Cheon, Kang, \& Hong, 2003). This disclosed ambiguity introduces by side lobes of timing metric.

To synchronize frequency offset, Morelli used a training symbol to cover the maximum estimation range (Morelli \& Mengali, 1999). Yet, the training symbol necessitated an exceptional design, implementing multiple identical sections to provide an adequate estimation range.

The challenge of subcarrier recovery includes channel estimation and phase estimation. William Shied proposed a method using pilot-aided (PA) and maximum-likelihood (ML) decision feedback (PA ML-DF) (Shieh, Tucker, Chen, $\mathrm{Yi}, \&$ Pendock, 2007). This process economizes bandwidth due to the fact that very few subcarriers are used in the form of pilot-aided for phase compensation. It demonstrates a reliable performance with a slight size of $\mathrm{CD}$. However, since the carrier frequency raises, the involvement of the $\mathrm{CD}$ is significant and the laser oscillator phase noise starts to become much higher. This degrades the efficiency of carrier frequency recovery and results in excessive phase jitter as well as cycle slip impacts in the receiver, thus requiring new and faster tracking schemes.

Local Oscillator (LO) laser phase misaligned is frequently estimated by cyclic prefix or preambles. This needs either a long cyclic prefix or short preamble spacing, but it considerably enhances the complexity of the transmitter since extra computations are required to be performed to get the $\mathrm{LO}$ offset estimate. Moreover, averaging of the estimates is required to improve the reliability, which diminishes the speed with which LO changes could be tracked.

This paper proposes for the first time, a new phase jitter synchronization based on a novel pilots' insertion approach, ML estimator and MMSE interpolators. The phase jitter synchronization as well as the phase RMSE performance of the proposed method is evaluated on a CO-OFDM system at $20 \mathrm{~Gb} / \mathrm{s}$ by numerical simulations. Simulation outcomes verify the effectiveness of the proposed synchronization technique and show better compensation performance.

This paper is organized as follows. Section II describes the system being considered. In Section III, the proposed synchronization method is presented. Section IV provides the simulation results, performance evaluation, and discussions. Finally, our conclusions are given in Section V.

\section{Simulation System Model}

In this research, we proposed a unique OFDM frame structure using pilot signal for phase noise synchronization. Pilots are disposed diagonally as presented in the Figure 1. Their positions are described by the following formula,

$$
p_{k, i}=p_{m N_{\mathrm{s}}+k, i}=\left\{\begin{array}{rr}
X_{k, i}, & i=k=2 m+1, \\
X_{k, N_{\mathrm{sb}}-k+1}, & k=2 m, i=N_{\mathrm{sb}}-k+1,
\end{array}\right.
$$

$m$ being an integer and $k \in\left[1 ; N_{\mathrm{s}}\right], i \in\left[1 ; N_{\mathrm{sb}}\right] . N_{\mathrm{s}}$ is the OFDM symbols numbers in one OFDM frame and $N_{\mathrm{sb}}$ the Number of subcarriers in one OFDM symbol.

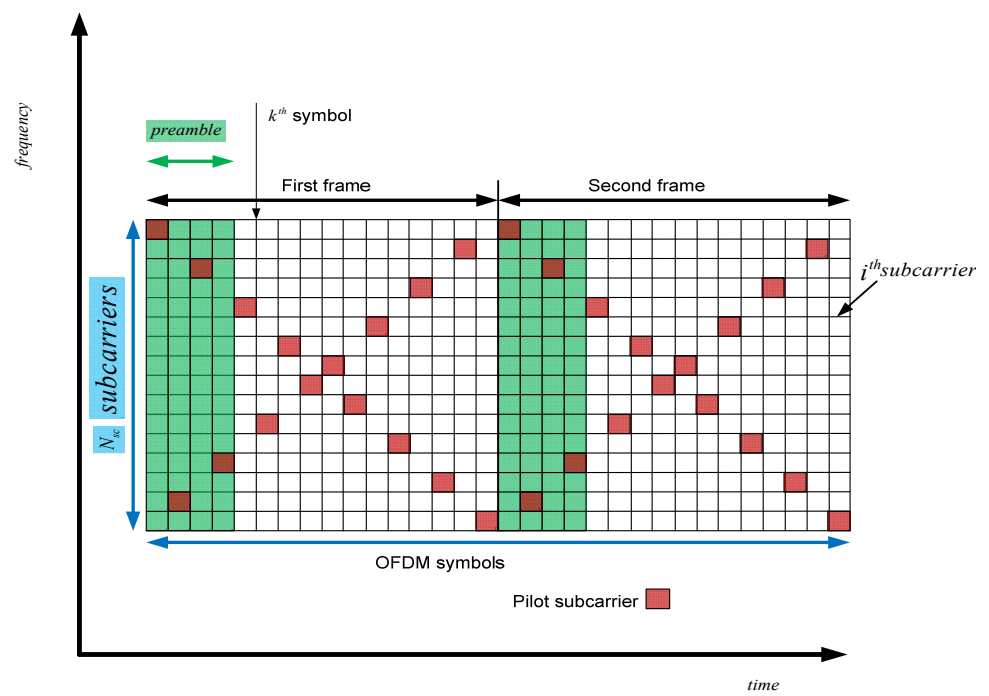

Figure 1. Data structure of CO-OFDM frame 
A conceptual diagram of our CO-OFDM simulation system is presented in Figure 2. As described in previous research (Minming et al., 2012), the received waveform in the frequency domain in the presence of oscillator laser phase noise is given by the following equations,

$$
R_{m}(k)=\theta_{0} H_{m}(k) X_{m}(k)+I_{m}(k)+N_{m}
$$

Where

$$
\begin{gathered}
\theta_{0}=\frac{1}{N} \mathrm{e}^{\mathrm{j}\left[\omega_{\mathrm{LD}}(t+\varepsilon)+\phi_{\mathrm{LD}}\right]} \sum_{n=0}^{N-1} \mathrm{e}^{\frac{\mathrm{j} 2 \pi l \varepsilon_{n}}{T}} \\
I_{m}=\sum_{k=0, k \neq m}^{N-1} H_{m}(k) X_{m}(k) \sum_{n=0}^{N-1} \theta_{k-m} \mathrm{e}^{\frac{\mathrm{j} 2 \pi}{N}(k-m) n}
\end{gathered}
$$

$\theta_{0}$ is the optical phase noise at the transmitter and the receiver including optical and radio frequency (RF) local oscillator phase noise.

$X_{m}(k)$ is the transmitted baseband OFDM signal.

$H_{m}(k)$ is the impulse response function of the fiber chromatic dispersion (CD).

$N_{m}$ is the noise that comes from the amplified spontaneous emission (ASE) noise of the optical amplifiers (OAs) in the optical fiber link. We do not consider nonlinearity and polarization mode dispersion effects in this paper.

Considering the assumptions of free inter-symbol interference (ISI) and perfect fast-Fourier-transform (FFT) window and frequency synchronization, Equation (2) can be written as follows,

$$
R_{m}(k)=\theta_{0} H_{m}(k) X_{m}(k)+N_{m}
$$

As shown in Figure 2, local oscillator phase noise synchronization happens at the receiver using Matlab component.

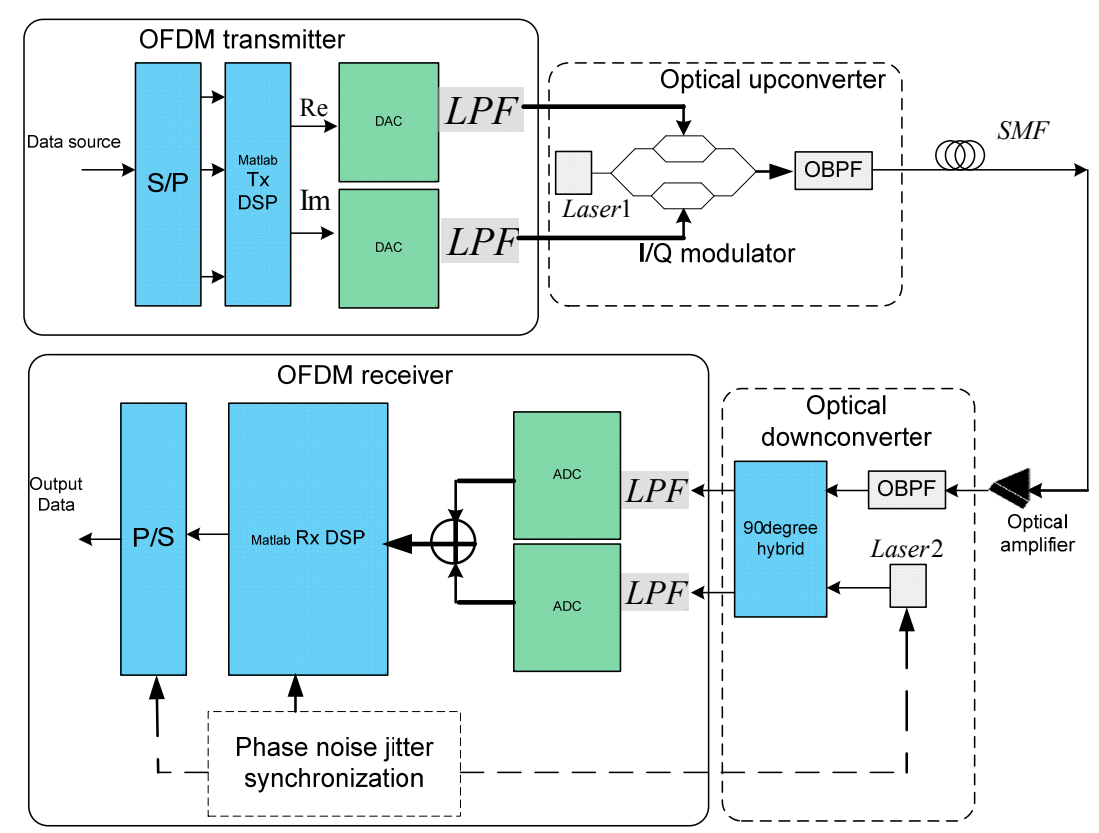

Figure 2. Simulation diagram of complete CO-OFDM with phase jitter noise synchronization S/P, serial-to-parallel; DAC, digital-to-analogue converter; LPF, low-pass filter; I/Q, inphase/quadrature; BPF, bandpass filter; OBPF, optical bandpass filter 


\section{Proposed Synchronization Scheme}

The proposed phase noise jitter synchronization used in this paper is illustrated in Figure 3. The proposed algorithm targets are optical coherent communication systems operating with a high level of transmitter/ receiver local lasers. The proposed synchronization algorithm makes use of regularly inserted pilot symbols which provide good carrier frequency and phase noise estimate with low jitter and no cycle slips. Square M-QAM constellations are used because they are easier to generate and are optimally immune against additive white Gaussian noise (AWGN) (Pfau, Hoffmann, \& Noé, 2009).

The synchronization is achieved in four steps: pilots' extraction, feed forward maximum likelihood (FF-ML) and linear interpolation, Wiener interpolation using the minimum mean square error (MMSE). The process presents carrier phase jitter synchronization based upon pilot data aided carrier of a received optical OFDM signal $R(n)$ bearing signal field consisted of $N_{\mathrm{P}}$ pilots of the pilot's set P, $N_{s}$ symbols of the symbol's set $S . N_{c}$ is the number of data subcarriers per symbol. $n, \ell$ are respectively the subcarrier index and symbol index.

The particularity is a couple of interpolation levels wherein the first is founded on Wiener filters applying the Minimum Mean Square Error (MMSE) and the following accomplishes the linear interpolation.

Figure 3 is an illustration of our synchronization technique associated with the following algorithm:

- Pilot signals extraction;

- Calculation of an unwrapped time phase estimate $\bar{\theta}(1 L)$ by Feed Forward Maximum Likelihood algorithm over the group of pilot subcarriers $\mathrm{P}$;

- $\quad$-Interpolation of mentioned unwrapped time estimates for consecutive signal symbols $(1,2, \ldots . ., \ell)$ with a wiener interpolator owning $\mathrm{M}$ taps to achieve interpolated phase estimates via Minimum Mean Square Error (MMSE);

- -Supply linear interpolation between mentioned interpolated phase estimates to achieve phase correction estimates $\bar{\theta}(1 L)$ over the data subcarriers;

- Calculation from mentioned phase correction estimate $\bar{\theta}(1 L)$ a phase correction $\left(e^{-j \bar{\theta}(\ell)}\right)$ to apply to mentioned OFDM signal $R(n)$.

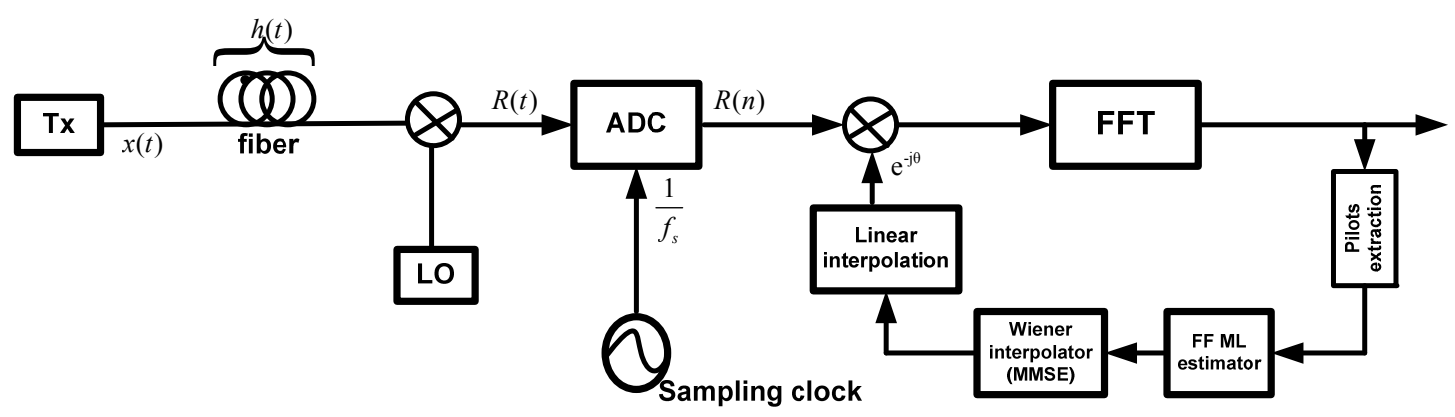

Figure 3. Carrier phase jitter synchronization technique structure in coherent optical OFDM receiver Tx, transceiver; LO, local oscillator; ADC, analog digital converter; FFT, fast fourier transform; FF-ML, feed forward maximum likelihood

This correction is going to be applied to the input signal $R(n)$ to achieve a phase corrected input signal for the data symbol signals. The pilot symbol signals do not ever necessitate being phase corrected, since they are eliminated at the input of the symbols de-mapper.

The four steps of synchronization, pilots' extraction, FF-ML, Wiener interpolation (MMSE) as well as linear interpolation are outlined in the next paragraphs.

\subsection{Pilots Extraction}

Pilots are extracted after FFT. They do not need being phase corrected, since they are eliminated at the output of the FFT. Timing recovery should preferably be ideally fixed and inter symbol interference is ignored. Physical layer frame alignment is supposed to be known to the receiver such that the pilot symbols could be extracted in their appropriate position. 


\subsection{Unwrapped Phase Estimation over Feed Forward Maximum Likelihood (FF-ML)}

To estimate the phase noise jitter, we resolved to employ Maximum Likelihood (ML) estimator because the transmit pilots symbol is known. In other hands, as the set of pilot $\mathrm{P}$ is short, from a few data to a few symbols, a Feed Forward (FF) method is recommended to improve the required estimation process. The maximum likelihood of phase noise jitter is provided by the following algebraic operation:

$$
\begin{gathered}
\bar{\theta}^{(p)}=\arg \left\{\sum_{k=0}^{N_{p}} C^{(p) *}(k) S^{(p)}(k)\right\} \\
C^{(p)^{*}}=\frac{1}{T_{s}} \int_{0}^{T_{s}} r\left(t-i T_{s}\right) S^{*}(k) d t
\end{gathered}
$$

designates the conjugate of $C^{(p)}(k)$ which designates the pilot carrier symbols that are overhead (they do not carry any information content).

Once a signal sample $S^{(p)}(k)$ corresponding to a pilot signal reaches the receiver, it's retrieved by the receiver and then sample by sample the above formula is computed.

In event the pilot symbols are small as well as the phase process quite slow, the time variation property of the carrier phase is mostly ignored. Under this hypothesis and with relatively high optical signal-to-noise ratio, it might be demonstrated that the ML estimated phase noise could be written as,

$$
\bar{\theta}^{(p)} \approx \theta+N_{I}
$$

$\theta$ designates the actual phase value. With $N_{I}$ being the zero-mean Gaussian noise contribution to phase estimate, with variance,

$$
\sigma_{N_{I}}^{2} \approx \sigma_{\theta}^{2}=\frac{1}{2 L_{p} E_{s} / N_{0}}
$$

which is independent from square M-QAM modulations constellations.

\subsection{Phase Noise Interpolation by Wiener Filters Using MMSE and Linear Interpolation}

The further step is the thoughtfulness of an interpolation technique where the interpolants in a given slot are computed with a higher order interpolation function. But the choice of the interpolation technique depends on the second effect of linear impairments such as frequency clock errors which would add a linear time varying term into the phase noise which is easily interpolated by Wiener filter using MMSE. That is why in this paper, we investigate on MMSE to derive an "optimum" interpolator. This kind of interpolation has already been used in wireless communications for fading channels $(\mathrm{Li}, 2000)$ to deal with fast varying fading process and somehow in optical communication (Ip \& Kahn, 2007). In that work a soft-decision phase-estimation stage followed by hard-decision estimation of the carrier phase and the transmitted symbols are used. The author modeled phase noise as a Wiener process and expressed that the optimal hard-decision phase estimator is a linear filter. The effect of a nonzero frequency offset between transmitter and LO lasers were investigated and found to be harmful to system performance. A numerical oscillator has been used to track the slow components of laser frequency drift. However the numerical oscillator component is another jitter source (Demir, 2006).

In our task we improved the approach of phase noise interpolation employing pilots structured as in Figure 1 then conducted over with the FF-ML phase estimation algorithm.

Wiener-Hopf equation is used to specify the coefficients for optimum linear estimation wherein simplifying assumptions are based on Wiener-Kolmogorov model for interpolations with $\mathrm{M}$ taps.

Since the pilots spacing is non-equidistant (see Figure 1), for convenient notation, we present here the vector $p$ which holds in all time indices $k \in K_{p}$ in addition to a vector $d$ that containing all time indices.

$$
k \in K_{d}: p=\left[p_{1}, \ldots, p_{k_{p}}\right]^{T}, d=\left[d_{1}, \ldots ., d_{k_{d}}\right]^{T}
$$

We considered the FF ML of estimated phase noise $\bar{\theta}^{(p)}$.

We derived a set of filters with coefficients $C_{k, \text { opt }}(m)$ (one filter for each symbol in time in one frame) such that the phase interpolants over the frame is: 


$$
\bar{\theta}\left(l L_{s}+k_{s}\right)=\sum_{m=-M}^{M} C_{k, o p t}(m) \bar{\theta}\left[(l+m) \cdot L_{s}\right]
$$

These interpolants are optimum in the sense that they minimize the mean square error (MSE).

$$
\varepsilon\left(k_{s}\right)=E\left\{\theta\left(l \cdot L_{s}+k_{s}\right)-\left.\sum_{m=-M}^{M} C_{k . o p t}(m) \bar{\theta}[(l+m) \cdot L]\right|^{2}\right\}
$$

Using the orthogonality principle (Cioffi, Dudevoir, Vedat, \& Forney Jr, 1995), the optimum coefficients are considered to be the solutions to the set of equations:

$$
E\left\{\left|\theta\left(l . L_{s}+k_{s}\right)-\bar{\theta}\left(l \cdot L_{s}+k_{s}\right)\right| \bar{\theta}^{*}(l+p) \cdot L_{s}\right\}=0
$$

for $p=0, \pm 1, \ldots, \pm M$ and $k_{s}=0,1 \ldots, L_{s}-1$.

Introducing the autocorrelation of $\bar{\theta}\left(l . L_{s}\right)$

$$
R_{\bar{\theta} \bar{\theta}}\left(p L_{s}\right)=E\left\{\bar{\theta}\left[(l+p) \cdot L_{s}\right] \bar{\theta}\left[l \cdot L_{s}\right]\right\}
$$

And the autocorrelation of $\theta\left(l . L_{s}\right)$ and $\bar{\theta}\left(l . L_{s}\right)$.

$$
R_{\theta \bar{\theta}}\left(p L_{s}\right)=E\left\{\theta\left[(l+p) \cdot L_{s}\right] \bar{\theta}\left[l \cdot L_{s}\right]\right\}
$$

Yielding to Wiener-Holf equations

$$
\begin{gathered}
\sum_{m=-M}^{M} C_{k . o p t}(m) R_{\bar{\theta} \bar{\theta}}\left(m L_{s}-p L_{s}\right)=R_{\theta \bar{\theta}}\left(k_{s}-p L_{s}\right) \\
p=0, \pm 1, \ldots, \pm M, \quad k_{s}=0,1, \ldots, L_{s}-1
\end{gathered}
$$

These equations can be written in matrix form as,

$$
R_{\hat{\theta} \hat{\theta}} C_{k, o p t}=r_{\theta \hat{\theta}_{k}}, k=0,1, \ldots, L_{s}-1
$$

Where

$$
\begin{gathered}
r_{\theta \bar{\theta}_{k}}=\left[R_{\theta \bar{\theta}}\left(k+M L_{s}\right), R_{\theta \bar{\theta}}\left(k+(M-1) L_{s}\right), \ldots, R_{\theta \bar{\theta}}(k), \ldots, R_{\theta \bar{\theta}}\left(k-M L_{s}\right)\right]^{T} \\
C_{k, p p t}=\left[C_{k, o p t}(-M), C_{k, \text { opt }}(-M+1), \ldots, C_{k, o p t}(0), \ldots, C_{k, \text { opt }}(M)\right]^{T}
\end{gathered}
$$

And

$$
\begin{aligned}
R_{\bar{\theta} \bar{\theta}} & =\left[\begin{array}{cccc}
R_{\bar{\theta} \bar{\theta}}\left(-M L_{s}+M L_{s}\right) & R_{\overline{\bar{\theta}} \bar{\theta}}\left((-M+1) L_{s}+M L_{s}\right) & \ldots & R_{\overline{\bar{\theta}} \bar{\theta}}\left(M L_{s}+M L_{s}\right) \\
R_{\bar{\theta} \bar{\theta}}\left(-M L_{s}+(M-1) L_{s}\right) & R_{\bar{\theta} \bar{\theta}}\left((-M+1) L_{s}+(M-1) L_{s}\right) & \ldots & R_{\bar{\theta}}\left(M L_{s}+(M-1) L_{s}\right) \\
\ldots & \ldots & \ldots & \ldots \\
R_{\bar{\theta} \bar{\theta}}\left(-M L_{s}-M L_{s}\right) & R_{\bar{\theta} \bar{\theta}}\left((-M+1) L_{s}-M L_{s}\right) & \ldots & R_{\bar{\theta} \bar{\theta}}\left(M L_{s}-M L_{s}\right)
\end{array}\right] \\
& =\left[\begin{array}{cccc}
R_{\bar{\theta} \bar{\theta}}(0) & R_{\bar{\theta} \bar{\theta}}\left(L_{s}\right) & \ldots & R_{\bar{\theta} \bar{\theta}}\left(2 M L_{s}\right) \\
R_{\bar{\theta} \bar{\theta}}\left(L_{s}\right) & R_{\bar{\theta} \bar{\theta}}(0) & \ldots & R_{\bar{\theta} \bar{\theta}}\left(2 M L_{s}-1\right) \\
\ldots & \ldots & \ldots & \ldots \\
R_{\bar{\theta} \bar{\theta}}\left(2 M L_{s}\right) & R_{\bar{\theta} \bar{\theta}}\left(2 M L_{s}-1\right) & \ldots & R_{\bar{\theta} \bar{\theta}}(0)
\end{array}\right]
\end{aligned}
$$

Earlier, it has been showed that the estimated value of phase noise may be written as,

$\bar{\theta}\left(l . L_{s}\right)=\theta\left(l . L_{s}\right)+N_{l}\left(l . L_{s}\right)$. Using this equation, autocorrelation and cross correlation functions can be put in the following forms,

$$
\begin{aligned}
& R_{\bar{\theta} \bar{\theta}}\left(p L_{s}\right)=R_{\theta \theta}(p L)+\frac{1}{2 L_{p} E_{s} / N_{0}} \delta(p) \\
& R_{\theta \bar{\theta}}\left(p L_{s}\right)=R_{\theta \theta}\left(p L_{s}\right)
\end{aligned}
$$

So the previous matrix formulation can be written as, 


$$
\left[R_{\theta \theta}+\frac{1}{2 L_{p} E_{s} / N_{0}} I\right] C_{k, o p t}=r_{\theta \theta_{k}}, k=0,1, \ldots, L_{s}-1
$$

This matrix can easily be solved by a matrix inversion for every $k=0,1,2, \ldots, L_{s}-1$.

$$
C_{k, \text { opt }}=\left[R_{\theta \theta}+\frac{1}{2 L_{p} E_{s} / N_{0}}\right]^{-1} r_{\theta \theta_{k}}, k=0,1, \ldots, L_{s}-1
$$

This equation means that the optimum coefficients (M taps) of Wiener filters rely on the autocorrelation function of the phase noise jitter and on the signal-to-noise ratio.

We can easily derive now the expression of the minimum value of mean square error as a result of the interpolation of the Wiener filter with previous coefficients $C_{k, o p t}$. Accomplishing this we rewrite the MSE equation matrix form, compute the expectations and then exploit the orthogonality principle to get,

$$
\begin{aligned}
& \varepsilon(k)=R_{\theta \theta}(0)-r_{\theta \theta_{k}}^{T} C_{k, o p t}=R_{\theta \theta}(0)-r_{\theta \theta_{k}}^{T}\left[R_{\theta \theta}+\frac{1}{2 L_{p} E_{s} / N_{0}}\right]^{-1} r_{\theta \theta_{k}} \\
& k=0,1, \ldots, L_{s}-1
\end{aligned}
$$

The average of the mean square error $\varepsilon(k)$ (in $d g^{2}$ unit) over the OFDM frame can be computed by:

$$
\bar{\varepsilon}=\frac{1}{L_{s}} \sum_{k=0}^{L_{s}-1} \varepsilon(k)
$$

The root mean square (RMSE) of phase error is given by,

$$
R M S E=\sqrt{\bar{\varepsilon}} \text { (degree) }
$$

\section{Simulations Results and Discussions}

Simulation parameters are summarized in Table 1 .

Table 1. CO-OFDM simulation system parameters

\begin{tabular}{ll}
\hline Parameter & Value \\
\hline Bit rate & $20 \mathrm{~Gb} / \mathrm{s}$ \\
\hline Transmission distance & $1000 \mathrm{~km}$ \\
\hline Average span loss & $16 \mathrm{~dB}$ \\
\hline Average dispersion D & $18 \mathrm{ps} / \mathrm{nm} / \mathrm{km}$ \\
\hline PDM coefficient & $0.07 \mathrm{ps} / \mathrm{km}$ \\
\hline OFDM symbols number & 1000 \\
\hline Subcarriers per symbol & 128 \\
\hline FFT points & 1024 \\
\hline Sampling rate & $20 \mathrm{GS} / \mathrm{s}$ (sampling period $1 /$ Ts $=0.05 \mathrm{~ns}$ ) \\
\hline Symbol period & $25.8 \mathrm{~ns}$ \\
\hline Guard time & $3.5 \mathrm{~ns}$ \\
\hline Filters taps numbers M & 20 \\
\hline
\end{tabular}

Figure 4 illustrates the OSNR versus phase jitter RMSE for different square QAM modulation schemes. We observe the superior performance of 4-QAM (or QPSK) compared to others modulation schemes. 


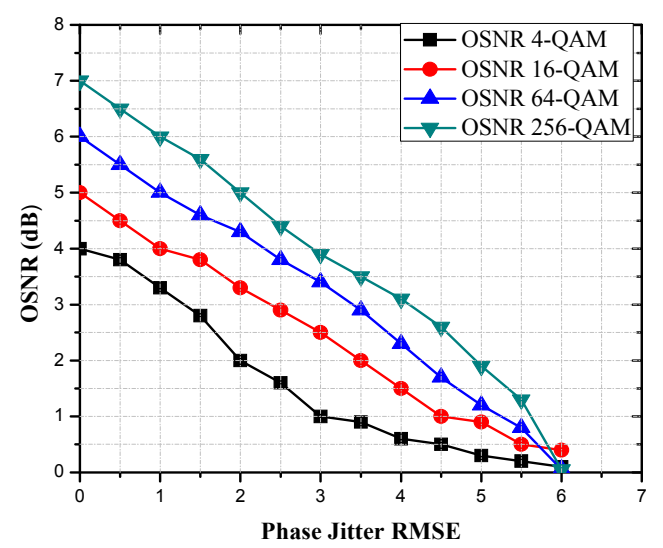

Figure 4. Influence of phase noise jitter on OSNR of square M-QAM

Table 2 shows the M-QAM phase jitter performance. We noticed that the RMSE attains a maximum phase jitter RMS (degree) of 5.75 degrees for 256-QAM then decreases to 3 degrees for 4-QAM. This reveals the better performance of 4-QAM compared to three others m-QAM schemes.

Table 2. Phase jitter RMSE requirements for different m-QAM to limit the performance degradation to 1-dB OSNR

\begin{tabular}{ll}
\hline Modulation constellation & Phase jitter RMS (degree) \\
\hline 4-QAM (QPSK) & 3 \\
16-QAM & 4.5 \\
\hline 64-QAM & 5.5 \\
\hline 256-QAM & 5.75 \\
\hline
\end{tabular}

Figure 5 displays the RMSE phase jitter of the PA-FF-ML algorithm for different OSNR values $(12 \mathrm{~dB}, 7 \mathrm{~dB}$ and $2 \mathrm{~dB}$ ). In other words, this figure is a comparison of different OSNR when PA-FF-ML algorithm is used. As observed the performance is undoubtedly far better for higher OSNR. It's moreover noted that the performance is relatively good in the case that the number of symbols increases above 1000.

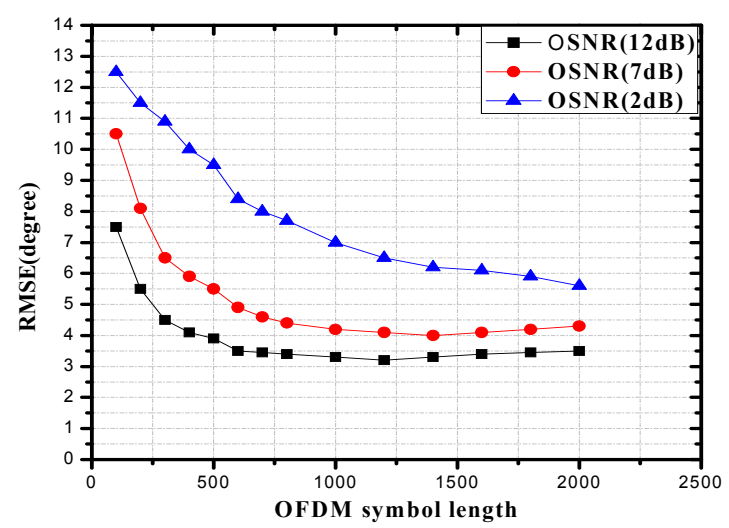

Figure 5. Root Mean Square Error (RMSE) as function of OFDM symbols length for different values of OSNR for PA FF ML

The following Figure 6 displays the simulations that had been run to access the efficiency of PA FF ML employing Wiener filters. In comparison with Figure 5, one could check that the efficiency improvements are really big for the entire set of analyzed symbols length. As an illustration, the RMSE of OSNR (12 dB) dotted line in Figure 6 is superior to the one in Figure 5 with very nearly 1 degree. 


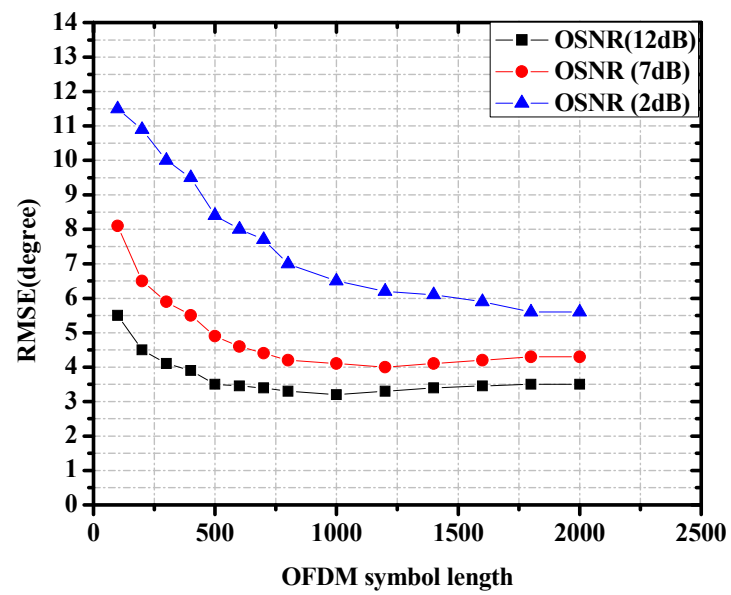

Figure 6. Root Mean Square Error (RMSE) as function of OFDM symbols length for different values of OSNR for PA FF ML using Wiener filters

Figure 7 displays the RMSE phase jitter of the synchronization algorithm for different values of OSNR with pilots' symbols length. As observed the performance is more effective for OSNR $=12 \mathrm{~dB}$ and then relatively invariable for every OSNR when the pilot symbols number gets to 20 .

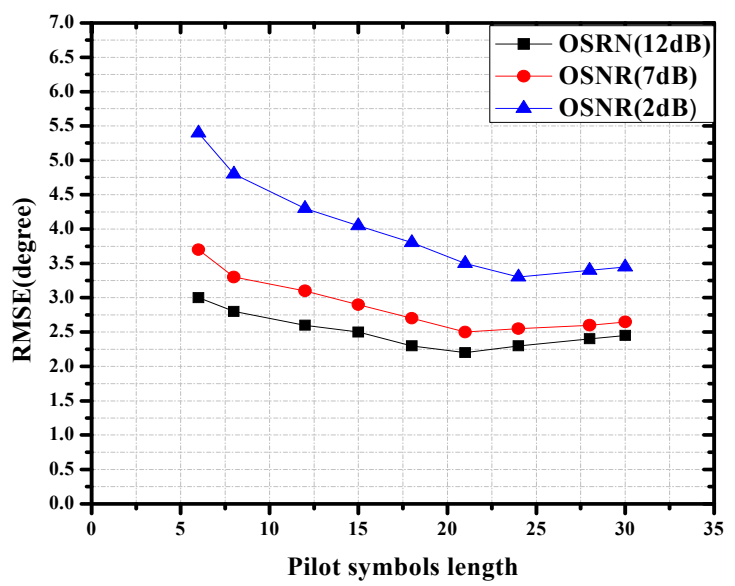

Figure 7. RMSE versus pilots symbols number $L_{p}$ for different OSNR values ( $2 \mathrm{~dB}, 7 \mathrm{~dB}$, and $\left.12 \mathrm{~dB}\right)$

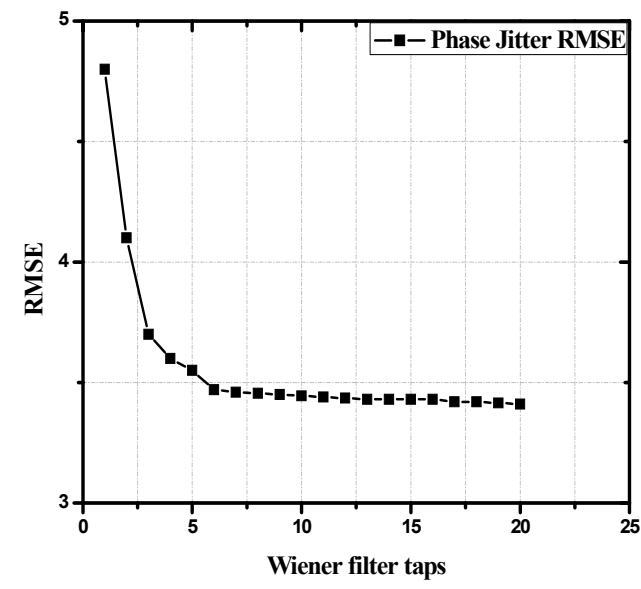

Figure 8. RMSE (degree) versus M filter taps 
This figure displays the dependency of the phase RMSE to the number of taps of the Wiener filter (2M+1). As certainly as we have observed, the RMSE decreases while M increases up to a place in which the phase noise is not more correlated at all so that there is not any positive aspects of the interpolation process. In this case, the RMSE function tends to be a flat line when the tap number attains 10. For this reason, the RMSE function could possibly be a flat line once the taps reaches a certain number. In Figure 8, this number could be 10 taps.

Figures 9 and 10 illustrate the RMSE of the phase jitter performance of the three distinct receivers' schemes (ideal receiver, PA-FF-ML and PA-FF-ML with MMSE) respectively for OSNR $=7 \mathrm{~dB}$ and $\mathrm{OSNR}=2 \mathrm{~dB}$. As observed in both cases, the performance begins to be moderately beneficial at very long symbols length (higher than 1000symbols). This indicates a relative sensitivity to sampling frequency errors.

Ideal receiver signifies the receiver is noise-free. It is apparent that it achieves much better. The principal purpose would be to do a comparison of PA-FF-ML and PA-FF-ML-MMSE. Figures 9 and 10 revealed outstanding efficiency of PA-FF-ML-MMSE in comparison with PA-FF-ML. One observed an effective performance of PA-FF-ML-MMSE in Figure 9. This is certainly because of the reason that OSNR is larger (7 dB) in Figure 9.

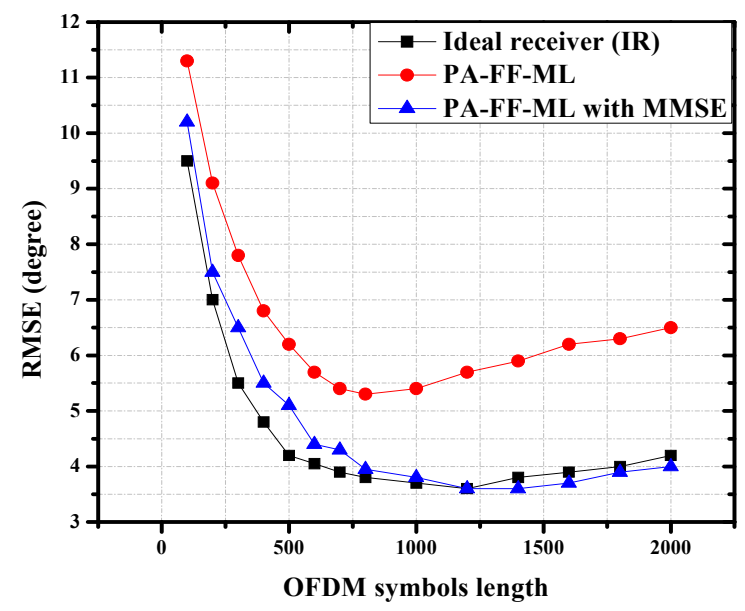

Figure 9. Comparison of free noise receiver (IR), pilot data aided feed forward maximum likelihood (PA FFML) and pilot data aided FFML employing wiener interpolators (MMSE) at OSNR $=7 \mathrm{~dB}$

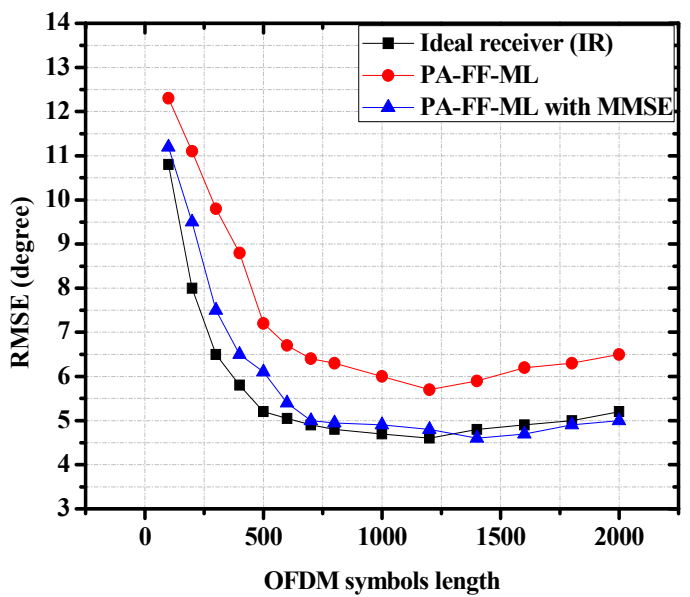

Figure 10. Comparison of free noise receiver (IR), pilot data aided feed forward maximum likelihood (PA FFML) and pilot data aided FFML with wiener interpolators (MMSE) at OSNR $=2 \mathrm{~dB}$

\section{Conclusion}

This paper proposed a unique carrier phase jitter synchronization technique based upon pilot data-aided feed forward maximum likelihood estimation (PA-FF-ML) and Wiener interpolator containing $\mathrm{M}$ taps to acquire 
interpolated phase estimates having a minimum mean square error interpolators (MMSE). Computations confirmed that the M taps coefficients and RMSE are a function of the autocorrelation of the phase as well as of the signal-to-noise ratio.

The simulation results confirmed that the synchronization approach offers better accurate phase jitter estimation. A comparison between four square QAM modulations, was produced, after which it had been verified that for $1 \mathrm{~dB}$ OSNR, 4-QAM achieves far better (3 degree) in comparison with 16-QAM (4.5 degree), 64-QAM (5.5 degree) and then 256-QAM (5.75 degree).

An evaluation of OSNR of PA-FF-ML method and PA-FF-ML which makes use of MMSE method, has proven the outstanding results of the last one. Somewhat far, the dependency of RMSE with pilots' symbol numbers and tap numbers has confirmed that the system performance might be good for no less than 20 pilots' symbol numbers and 10 tap numbers. By the end a study of the three various receiver schemes is presented for $7 \mathrm{~dB}$ and 2dB OSNRs. Once more PA-FF-ML using MMSE has proven an appropriate efficiency.

\section{Acknowledgements}

This work was supported by the National Natural Science Foundation of China. The authors also acknowledge Dr. Deng Lei at National Engineering Laboratory for Next Generation Internet Access System, School of Optical and Electronics Information Engineering, Huazhong University of Science and Technology, for the numerous suggestions and guidance for the completion of this work.

\section{References}

Cioffi, J. M., Dudevoir, G. P., Vedat, E. M., \& Forney Jr, G. D. (1995). MMSE decision-feedback equalizers and coding. I. Equalization results. Communications, IEEE Transactions on, 43(10), 2582-2594. $\mathrm{http}: / / \mathrm{dx}$. doi.org/10.1109/26.469441

Demir, A. (2006). Computing Timing Jitter From Phase Noise Spectra for Oscillators and Phase-Locked Loops With White and 1/f noise. Circuits and Systems I: Regular Papers, IEEE Transactions on, 53(9), 1869-1884. http://dx.doi.org/10.1109/TCSI.2006.881184

Ip, E., \& Kahn, J. M. (2007). Feedforward carrier recovery for coherent optical communications. Journal of Lightwave Technology, 25(9), 2675-2692. http://dx.doi.org/10.1109/JLT.2007.902118

Li, Y. (2000). Pilot-symbol-aided channel estimation for OFDM in wireless systems. Vehicular Technology, IEEE Transactions on, 49(4), 1207-1215. http://dx.doi.org/10.1109/25.875230

Minming, Z., Deming, L., \& He, W. (2012). Performance Analysis of Coherent Optical OFDM with Weiner Phase Noise jitters. Paper presented at the Information Optoelectronics, Nanofabrication and Testing.

Minn, H., Bhargava, V. K., \& Letaief, K. B. (2003). A robust timing and frequency synchronization for OFDM systems. Wireless Communications, IEEE Transactions on, 2(4), 822-839. http://dx.doi.org/10.1109/TWC.2003.814346

Morelli, M., \& Mengali, U. (1999). An improved frequency offset estimator for OFDM applications. Paper presented at the Communication Theory Mini-Conference, 1999.

Park, B., Cheon, H., Kang, C., \& Hong, D. (2003). A novel timing estimation method for OFDM systems. Communications Letters, IEEE, 7(5), 239-241. http://dx.doi.org/10.1109/LCOMM.2003.812181

Pfau, T., Hoffmann, S., \& Noé, R. (2009). Hardware-Efficient Coherent Digital Receiver Concept With Feedforward Carrier Recovery for \$ M \$-QAM Constellations. Journal of Lightwave Technology, 27(8), 989-999. http://dx.doi.org/10.1109/JLT.2008.2010511

Pollet, T., Van Bladel, M., \& Moeneclaey, M. (1995). BER sensitivity of OFDM systems to carrier frequency offset and Wiener phase noise. Communications, IEEE Transactions on, 43(234), 191-193. http://dx.doi.org/10.1109/26.380034

Schmidl, T. M., \& Cox, D. C. (1997). Robust frequency and timing synchronization for OFDM. Communications, IEEE Transactions on, 45(12), 1613-1621. http://dx.doi.org/10.1109/26.650240

Shieh, W. (2008). Maximum-likelihood phase and channel estimation for coherent optical OFDM. Photonics Technology Letters, IEEE, 20(8), 605-607. http://dx.doi.org/10.1109/LPT.2008.918873

Shieh, W., \& Athaudage, C. (2006). Coherent optical orthogonal frequency division multiplexing. Electronics Letters, 42(10), 587-589. http://dx.doi.org/10.1049/el:20060561

Shieh, W., \& Djordjevic, I. (2009). OFDM for optical communications: Access Online via Elsevier. 
Shieh, W., Tucker, R. S., Chen, W., Yi, X., \& Pendock, G. (2007). Optical performance monitoring in coherent optical OFDM systems. Opt. Express, 15(2), 350-356. http://dx.doi.org/10.1364/OE.15.000350

\section{Copyrights}

Copyright for this article is retained by the author(s), with first publication rights granted to the journal.

This is an open-access article distributed under the terms and conditions of the Creative Commons Attribution license (http://creativecommons.org/licenses/by/3.0/). 